\title{
Reliability test plan based on Dagum distribution
}

\author{
Bander Al-Zahrani \\ Department of Statistics, King Abdulaziz University, Jeddah, Saudi Arabia
}

E-mail: bmalzahrani@kau.edu.sa

\begin{abstract}
Apart from other probability models, Dagum distribution is also an effective probability distribution that can be considered for studying the lifetime of a product/material. Reliability test plans deal with sampling procedures in which items are put to test to decide from the life of the items to accept or reject a submitted lot. In the present study, a reliability test plan is proposed to determine the termination time of the experiment for a given sample size, producers risk and termination number when the quantity of interest follows Dagum distribution. In addition to that, a comparison between the proposed and the existing reliability test plans is carried out with respect to time of the experiment. In the end, an example illustrates the results of the proposed plan.
\end{abstract}

Keywords: Acceptance sampling plan; Consumer and Producer's risks; Dagum distribution; Truncated life test

\section{Introduction}

Dagum [1] introduced Dagum distribution as an alternative to the Pareto and log-normal models for modeling personal income data. This distribution has been extensively used in various fields such as income and wealth data, meteorological data, and reliability and survival analysis. The Dagum distribution is also known as the inverse Burr XII distribution, especially in the actuarial literature. An important characteristic of Dagum distribution is that its hazard function can be monotonically decreasing, an upside-down bathtub, or bathtub and then upside down bathtub shaped, for details see Domma [2]. The behavior thus discussed led several authors to study the model in different fields. Of late Dagum distribution has been investigated from a reliability point of view and used to analyze survival data (see Domma et al., [3], [4]). Kleiber and Kotz [5] and Kleiber [6] provided an exhaustive review on the origin of the Dagum model and its applications. Domma et al. [3] estimated the parameters of Dagum distribution with censored samples. Shahzad and Asghar [7] used TL-moments to estimate the parameters of this distribution. Oluyede and Ye [8] presented the class of weighted Dagum and related distributions. Domma and Condino [4] proposed the five parameter beta-Dagum distribution.

A continuous random variable $T$ is said to have a three-parameter Dagum distribution, abbreviated as $T \sim \operatorname{Dag}(\beta, \sigma, \delta)$, if its density probability function (pdf) is given as

$$
f(t ; \beta, \sigma, \delta)=\frac{\beta \delta}{\sigma}\left(\frac{t}{\sigma}\right)^{-\delta-1}\left(1+\left(\frac{t}{\sigma}\right)^{-\delta}\right)^{-\beta-1}, t>0,
$$

where $\sigma>0$ is the scale parameter and its two shape parameters $\beta$ and $\delta$ are both positive. The corresponding distribution function of
(1) is given by

$$
F(t ; \beta, \sigma, \delta)=\left(1+\left(\frac{t}{\sigma}\right)^{-\delta}\right)^{-\beta}, t>0, \beta, \sigma, \delta>0 .
$$

Further probabilistic properties of this distribution are given, for example, in Dagum [1].

Acceptance sampling plans based on truncated life tests for exponential distribution was first discussed by Epstein [9], see also Sobel and Tischendrof [10]. The results were extended for the Weibull distribution by Goode and Kao [11]. Gupta and Groll [12] and Gupta [13] provided extensive tables on acceptance sampling plans for gamma, normal and log-normal distributions. Kantam and Rosaiah [14], Kantam et al. [15], Rosaiah and Kantam [16], Balakrishnan et al. [17] and Aslam and Shahbaz [18] provide the time truncated acceptance plans for half-logistics, log-logistics, Rayleigh, generalized Birnbaum-Saunders and generalized exponential distributions respectively.

The primary purpose of this study is to develop reliability test plans to decide whether to accept or reject a submitted lot of products whose lifetime is assumed to be a Dagum distribution. Section 2 describes the use of reliability test plans and how it can be profitably used for making decisions. An illustrative numerical example of ordered failure times is given in section 2 . We conclude in section 3 .

\section{Reliability test plans}

Acceptance sampling, if applied to a series of lots, prescribes a procedure that will give a specified probability of accepting lots of given quality. It is the process of inspecting a sample of product/material drawn from the lot to accept or reject the lot as either conforming to or not conforming to quality specifications i.e. inspection based on qualitative measurements. Inspection for acceptance purpose is used 
at various stages in manufacturing process. There are two ways in which inspection is carried out: (i) $100 \%$ inspection. (ii) Sampling inspection. Sampling inspection can be defined as a technique to determine the acceptance or rejection of a lot or population by some defective parts found in a random sample drawn from the lot. If the number of defective items does not exceed a predefined level, the lot is accepted. Otherwise, it is rejected.

In acceptance sampling inspection a defective article is defined as one that fails to conform to specifications in one or more quality characteristics. A common procedure in acceptance sampling is to consider each submitted lot of product separately and to base the decision on acceptance or rejection of the lot on the evidence of one or more samples chosen at random from the lot. If the quality of interest under consideration is the lifetime of the product that is put for testing, then the sample of lifetimes of the sampled products is the output that we obtain after the completion of sampling inspection If good products are rejected by sample information, this error is called type-1 error. Whereas, if the good products are not accepted by the consumer, this error is called a type- 2 error. Moreover, if a decision to accept or reject the lot is subjected to the risks associated with the two types of errors; this procedure is statistically termed as 'acceptance sampling based on life tests' or 'reliability test plans. To compare the performance of various acceptance sampling plans, their performance over a range of possible quality levels is studied. In statistical quality control, acceptance sampling plan is concerned with the inspection of a sample of products taken from a lot and the decision whether to accept or not to accept the lot based on the quality of the product. Here we discuss the reliability test plan for accepting or rejecting a lot where the lifetime of the product follows Dagum distribution. In a life testing experiment, the procedure is to terminate the test by a predetermined time $t$ and note the number of failures. If the number of failures at the end of time $t$ does not exceed a given number $c$, called acceptance number then we accept the lot with a given probability of at least $p$. But if the number of failures exceeds $c$ before time $t$ then the test is terminated, and the lot is rejected. For such truncated life test and the associated decision rule, we are interested in obtaining the smallest sample size to arrive at a decision.

In the sequel, we assume that the distribution parameters $\beta$ and $\delta$ are known, while $\sigma$ is unknown. In such a case, the average lifetime of the product depend only on $\sigma$, and can be observed that the average lifetime is monotonically increasing in $\sigma$. Let $\sigma_{0}$ represent the required minimum average lifetime, then, for given values of $\beta$ and $\delta$.

The consumer's risk, i.e., the probability of accepting a bad lot should not exceed $1-p^{*}$, where $p^{*}$ is a lower bound for the probability that a lot of true value of $\sigma$ below $\sigma_{0}$ is rejected by the sampling plan. For a fixed $p^{*}$, sampling plan is characterized by $\left(n, c, t / \sigma_{0}\right)$. By sufficiently large lots we can apply binomial distribution to find acceptance probability. The problem is to determine the smallest positive integer $n$, for given values of $p^{*}, \sigma_{0}$ and $c$, such that

$$
L\left(p_{0}\right)=\sum_{i=0}^{c}\left(\begin{array}{l}
n \\
i
\end{array}\right) p_{0}^{i}\left(1-p_{0}\right)^{n-i} \leq 1-p^{*},
$$

where $p_{0}=F\left(t ; \beta, \delta, \sigma_{0}\right)$, obtained from equation (2), indicates the failure probability before time $t$ and depends only on the ratio $t / \sigma_{0}$. The function $L(p)$ is the operating characteristic function of the sampling plan, i.e. the acceptance probability of the lot as function of the failure probability $p(\sigma)=F(t ; \beta, \delta, \sigma)$. The average lifetime of the products is increasing in $\sigma$ and, therefore, the failure probability $p(\sigma)=F(t ; \beta, \delta, \sigma)$ is decreasing function in $\sigma$ which implies that the operating characteristic function is increasing in $\sigma$. The minimum values of $n$ satisfying the inequality (3) are obtained and displayed in Table 2 for $p^{*}=0.90,0.95,0.99$ and $t / \sigma_{0}=$ $0.628,0.942,1.257,1.571,2.356,3.141,3.927,4.712$ for $\beta=\delta=2$. If $p=F(t ; \beta, \delta, \sigma)$ is small and $n$ is large, the binomial probability may be approximated by Poisson probability with parameter $n p$ so that the left side of (3) can be written as

$$
L^{*}(p)=\sum_{i=0}^{c} \frac{(n p)^{i}}{i !} e^{-n p} \leq 1-p^{*}
$$

The minimum values of $n$ satisfying (4) are obtained for the same combination of $p^{*}$ and $t / \sigma_{0}$ values as those used for (3). The results are given in Table 3

Alternatively, Kantam et al. (2006) considered another approach for a reliability test plan. We summarize this approach. Let $n$ indicates the number of sampled items to be determined and $r$ stands for a natural number, such that if $r$ failures out of $n$ samples are occured before the terminated time $t$ the lot would be rejected. In this aspect, $r$ is called as termination number. The sample size is depending upon the cost consideration and the expected time to reach a decision. If the sample size is large it may reduce the expected waiting time but increases the cost of consideration. Let us take sample size as a multiple of the termination number to balance between these two aspects. As we have to come know that the probability of $r$ failures out of $n$ tested items is given as ${ }_{n} C_{r} p^{r}(1-p)^{n-r}$, where $p=F(t ; \beta, \delta, \sigma)$ as before. Thus, the probability of accepting the lot is

$$
L(p)=\sum_{i=0}^{r-1}\left(\begin{array}{l}
n \\
i
\end{array}\right) p^{i}(1-p)^{n-i} .
$$

If $\alpha$ is producer's risk then equation (6) can be written as:

$$
\sum_{i=0}^{r-1}\left(\begin{array}{l}
n \\
i
\end{array}\right) p^{i}(1-p)^{n-i}=1-\alpha
$$

Given the values of $n=r k, r$ and $k$, equation (6) can be solved for $p$ using cumulative probabilities of binomial distribution. Then the values of $p$ can be used in equation (6) for $\alpha=0.10,0.05,0.01$ to find the values of $t / \sigma$. These values for different values of $r$ and $n$ are given in Table 1.

As an example of this approach, let us think that we have to derive a life test sampling plan with an acceptance probability of 0.95 for lots with an acceptable mean life of 1000 hours and 10, 5 as sample size, termination number $r$ respectively. From Table 1, the entry against $r=5$ under column $2 r$ is 0.94479 . This implies that the termination time $t=944.79$ hours. In this test plan, we select 10 items from the submitted lot and put to test. We reject the lot, when the 5th failure is occured before 944.79 hours, otherwise we accept the lot. In either case terminating the experiment as soon as the 5th failure occurs or the termination time 944.79 hours is reached, or whichever is earlier.

\section{Concluding Remarks}

In this paper reliability test plan under the assumption that the life of a product follows a Dagum distribution is proposed. Values of termination time for Dagum distribution were provided so that the practitioners can use the suggested plan conveniently. Minimum sample size required to accept or reject a submitted lot for a given acceptance number with producer's risk using Binomial and Poisson approximation were obtained. It is safe to conclude that the proposed plan is useful in minimizing the producer's risk.

\section{References}

[1] C. Dagum, A New Model for Personal Income Distribution: Specification and Estimation, Economic Applique, Vol. 33, (1977), pp. 413-437.

[2] F. Domma, L'andamento della Hazard function nel modello di Dagum a tre parametri, Quaderni di Statistica, Vol. 4, (2002), pp. 103-114.

[3] F. Domma S. Giordano and M. M. Zenga, Maximum likelihood estimation in Dagum distribution with censored sample, Journal of Applied Statistics, Vol. 38, (2011), pp. 2971-2985. 
Table 1: Reliability test plan for Dagum distribution for $\beta=\delta=2$

\begin{tabular}{|c|c|c|c|c|c|c|c|c|c|}
\hline \multirow[b]{2}{*}{$r \backslash n$} & \multicolumn{9}{|c|}{$\alpha=0.10$} \\
\hline & $2 r$ & $3 r$ & $4 r$ & $5 r$ & $6 r$ & $7 r$ & $8 r$ & $9 r$ & $10 r$ \\
\hline 1 & 0.54118 & 0.47766 & 0.43844 & 0.41082 & 0.38986 & 0.37315 & 0.35939 & 0.34775 & 0.33772 \\
\hline 2 & 0.77885 & 0.66135 & 0.59578 & 0.55196 & 0.51977 & 0.49471 & 0.47440 & 0.45745 & 0.44300 \\
\hline 3 & 0.90130 & 0.74977 & 0.66915 & 0.61654 & 0.57846 & 0.54910 & 0.52549 & 0.50590 & 0.48928 \\
\hline 4 & 0.97932 & 0.80408 & 0.71350 & 0.65521 & 0.61337 & 0.58131 & 0.55564 & 0.53441 & 0.51644 \\
\hline 5 & 1.03466 & 0.84172 & 0.74393 & 0.68159 & 0.63710 & 0.60314 & 0.57602 & 0.55365 & 0.53474 \\
\hline 6 & 1.07656 & 0.86977 & 0.76646 & 0.70103 & 0.65454 & 0.61915 & 0.59095 & 0.56772 & 0.54812 \\
\hline 7 & 1.10971 & 0.89170 & 0.78398 & 0.71612 & 0.66805 & 0.63153 & 0.60248 & 0.57858 & 0.55844 \\
\hline 8 & 1.13680 & 0.90946 & 0.79811 & 0.72825 & 0.67890 & 0.64147 & 0.61172 & 0.58729 & 0.56670 \\
\hline 9 & 1.15948 & 0.92421 & 0.80981 & 0.73829 & 0.68785 & 0.64966 & 0.61935 & 0.59446 & 0.57350 \\
\hline \multirow[t]{2}{*}{10} & 1.17882 & 0.93671 & 0.81971 & 0.74676 & 0.69541 & 0.65657 & 0.62577 & 0.60050 & 0.57923 \\
\hline & \multicolumn{9}{|c|}{$\alpha=0.05$} \\
\hline 1 & 0.43501 & 0.38690 & 0.35671 & 0.33523 & 0.31881 & 0.30566 & 0.29477 & 0.28554 & 0.27756 \\
\hline 2 & 0.67409 & 0.57843 & 0.52393 & 0.48709 & 0.45981 & 0.43845 & 0.42106 & 0.40650 & 0.39405 \\
\hline 3 & 0.80187 & 0.67443 & 0.60521 & 0.55953 & 0.52621 & 0.50039 & 0.47953 & 0.46217 & 0.44740 \\
\hline 4 & 0.88498 & 0.73461 & 0.65531 & 0.60372 & 0.56644 & 0.53773 & 0.51464 & 0.49549 & 0.47924 \\
\hline 5 & 0.94479 & 0.77688 & 0.69012 & 0.63424 & 0.59410 & 0.56331 & 0.53863 & 0.51821 & 0.50092 \\
\hline 6 & 0.99060 & 0.80870 & 0.71612 & 0.65692 & 0.61459 & 0.58222 & 0.55633 & 0.53496 & 0.51688 \\
\hline 7 & 1.02718 & 0.83378 & 0.73649 & 0.67463 & 0.63055 & 0.59693 & 0.57009 & 0.54795 & 0.52925 \\
\hline 8 & 1.05730 & 0.85421 & 0.75301 & 0.68896 & 0.64344 & 0.60879 & 0.58116 & 0.55841 & 0.53920 \\
\hline 9 & 1.08267 & 0.87127 & 0.76676 & 0.70085 & 0.65413 & 0.61861 & 0.59033 & 0.56706 & 0.54743 \\
\hline \multirow[t]{2}{*}{10} & 1.10443 & 0.88581 & 0.77843 & 0.71094 & 0.66318 & 0.62692 & 0.59808 & 0.57436 & 0.55437 \\
\hline & \multicolumn{9}{|c|}{$\alpha=0.01$} \\
\hline 1 & 0.27603 & 0.24775 & 0.22964 & 0.21660 & 0.20653 & 0.19842 & 0.19166 & 0.18591 & 0.18092 \\
\hline 2 & 0.50770 & 0.44226 & 0.40382 & 0.37736 & 0.35754 & 0.34188 & 0.32904 & 0.31823 & 0.30895 \\
\hline 3 & 0.64078 & 0.54804 & 0.49600 & 0.46101 & 0.43519 & 0.41500 & 0.39858 & 0.38484 & 0.37309 \\
\hline 4 & 0.73022 & 0.61664 & 0.55476 & 0.51378 & 0.48382 & 0.46054 & 0.44171 & 0.42600 & 0.41262 \\
\hline 5 & 0.79601 & 0.66586 & 0.59642 & 0.55092 & 0.51787 & 0.49232 & 0.47171 & 0.45458 & 0.44001 \\
\hline 6 & 0.84724 & 0.70346 & 0.62797 & 0.57890 & 0.54343 & 0.51610 & 0.49412 & 0.47588 & 0.46040 \\
\hline 7 & 0.88869 & 0.73345 & 0.65296 & 0.60097 & 0.56354 & 0.53477 & 0.51168 & 0.49255 & 0.47634 \\
\hline 8 & 0.92319 & 0.75811 & 0.67340 & 0.61896 & 0.57989 & 0.54993 & 0.52592 & 0.50606 & 0.48923 \\
\hline 9 & 0.95254 & 0.77888 & 0.69054 & 0.63400 & 0.59353 & 0.56256 & 0.53777 & 0.51729 & 0.49995 \\
\hline 10 & 0.97791 & 0.79669 & 0.70517 & 0.64681 & 0.60514 & 0.57329 & 0.54783 & 0.52681 & 0.50904 \\
\hline
\end{tabular}

[4] F. Domma and F. Condino, The beta-Dagum distribution: definition and properties, Communications in Statistics-Theory and Methods, Vol. 42, (2013), pp. 4070-4090.

[5] C. Kleiber and S. Kotz, Statistical Size Distributions in Economics and Actuarial Sciences. John Wiley, Hoboken, New Jersey, 2003.

[6] C. Kleiber, A guide to the Dagum distributions. Springer, New York, 2008.

[7] M. N. Shahzad and Z. Asghar, Comparing TL-Moments, L-Moments and Conventional Moments of Dagum Distribution by Simulated data, Revista Colombiana de Estadistica, Vol. 36, (2013), pp. 79-93.

[8] B. O. Oluyede and Y. Ye, Weighted Dagum and related distributions, Afrika Matematika, Vol. 25, (2014), pp. 1125-1141.

[9] B. Epstein, Truncated life test in the exponential case, Annals of Mathematical Statistics, Vol. 25, (1954), pp. 555-564.

[10] M. Sobel, and J. A. Tischendrof, Acceptance sampling with new life test objectives, Proceedings of the Fifth National Symposium on Relia bility and Quality Control, Philadelphia, (1959), pp. 108-118.

[11] H. P. Goode, and J. H. K. Kao, Sampling plans based on the Weibull distribution, Proceedings of the Seventh National Symposium on Reliability and Quality Control, Philadelphia, (1961), pp. 24-40.

[12] S. S. Gupta, and P. A. Groll, Gamma distribution in acceptance sampling based on life tests, Journal of the American Statistical Association, Vol. 56, (1961), pp. 942-970.

[13] S. S. Gupta, Life test plans for normal and log-normal distributions, Technometrics, Vol. 4, (1962), pp. 151-160.

[14] R. R. L. Kantam, and K. Rosaiah, Half-logistic distribution in acceptance sampling based on life tests, APQR Transactions, Vol. 23, (1998), pp. 117-125.

[15] R. R. L. Kantam, K. Rosaiah, and R. C. Srinivas, Acceptance sampling plans based based on life tests: Log-logistic model, Journal of Applied Statistics, Vol. 28, (2001), pp. 121-128.

[16] K. Rosaiah, and R. R. L. Kantam, Acceptance sampling plans based based on inverse Rayleigh distribution, Economic Quality Control, Vol. 20, (2001), pp: 151-160.
[17] N. Balakrishnan, V. Leiva, and J. Lopez, Acceptance sampling plans from truncated life test based on generalized Birnbaum-Saunders distribution, Communications in Statistics-Simulation and Computation, Vol. 36, (2007), pp: 643-656.

[18] M. Aslam, M.Q. Shahbaz, Economic reliability tests plans using the generalized exponential distribution, Journal of Statistics, Vol. 14, (2007), pp: 52-59. 
Table 2: Minimum sample size required to accept/reject a submitted lot for a given acceptance number with producer's risk $p^{*}$ using Binomial approximation

\begin{tabular}{|c|c|c|c|c|c|c|c|c|c|}
\hline \multirow[b]{2}{*}{$1-p^{*}$} & \multirow[b]{2}{*}{$r$} & \multicolumn{7}{|c|}{$t / \sigma$} & \multirow[b]{2}{*}{4.712} \\
\hline & & 0.628 & 0.942 & 1.257 & 1.571 & 2.356 & 3.141 & 3.927 & \\
\hline \multirow[t]{11}{*}{0.90} & 0 & 28 & 10 & 5 & 4 & 2 & 2 & 2 & 1 \\
\hline & 1 & 48 & 17 & 9 & 7 & 4 & 3 & 3 & 3 \\
\hline & 2 & 65 & 23 & 13 & 9 & 6 & 5 & 4 & 4 \\
\hline & 3 & 82 & 29 & 16 & 12 & 8 & 6 & 6 & 5 \\
\hline & 4 & 98 & 35 & 20 & 14 & 9 & 8 & 7 & 6 \\
\hline & 5 & 114 & 40 & 23 & 16 & 11 & 9 & 8 & 8 \\
\hline & 6 & 130 & 46 & 26 & 19 & 12 & 10 & 9 & 9 \\
\hline & 7 & 145 & 51 & 29 & 21 & 14 & 12 & 11 & 10 \\
\hline & 8 & 160 & 57 & 32 & 23 & 15 & 13 & 12 & 11 \\
\hline & 9 & 175 & 62 & 36 & 26 & 17 & 14 & 13 & 12 \\
\hline & 10 & 190 & 67 & 39 & 28 & 19 & 16 & 14 & 13 \\
\hline \multirow[t]{11}{*}{0.95} & 0 & 36 & 12 & 7 & 5 & 3 & 2 & 2 & 2 \\
\hline & 1 & 58 & 20 & 11 & 8 & 5 & 4 & 3 & 3 \\
\hline & 2 & 77 & 27 & 15 & 11 & 7 & 5 & 5 & 4 \\
\hline & 3 & 95 & 33 & 19 & 13 & 8 & 7 & 6 & 6 \\
\hline & 4 & 112 & 39 & 22 & 16 & 10 & 8 & 7 & 7 \\
\hline & 5 & 129 & 45 & 26 & 18 & 12 & 10 & 9 & 8 \\
\hline & 6 & 146 & 51 & 29 & 21 & 13 & 11 & 10 & 9 \\
\hline & 7 & 162 & 57 & 32 & 23 & 15 & 12 & 11 & 10 \\
\hline & 8 & 178 & 62 & 35 & 25 & 17 & 14 & 12 & 12 \\
\hline & 9 & 193 & 68 & 39 & 28 & 18 & 15 & 14 & 13 \\
\hline & 10 & 209 & 74 & 42 & 30 & 20 & 16 & 15 & 14 \\
\hline \multirow[t]{11}{*}{0.99} & 0 & 56 & 19 & 10 & 7 & 4 & 3 & 3 & 2 \\
\hline & 1 & 81 & 28 & 15 & 10 & 6 & 5 & 4 & 4 \\
\hline & 2 & 102 & 35 & 19 & 13 & 8 & 7 & 6 & 5 \\
\hline & 3 & 122 & 42 & 23 & 16 & 10 & 8 & 7 & 6 \\
\hline & 4 & 142 & 49 & 27 & 19 & 12 & 10 & 8 & 8 \\
\hline & 5 & 160 & 56 & 31 & 22 & 14 & 11 & 10 & 9 \\
\hline & 6 & 178 & 62 & 35 & 24 & 15 & 13 & 11 & 10 \\
\hline & 7 & 196 & 68 & 38 & 27 & 17 & 14 & 12 & 12 \\
\hline & 8 & 213 & 74 & 42 & 30 & 19 & 15 & 14 & 13 \\
\hline & 9 & 230 & 80 & 45 & 32 & 21 & 17 & 15 & 14 \\
\hline & 10 & 247 & 86 & 49 & 34 & 22 & 18 & 16 & 15 \\
\hline
\end{tabular}

Table 3: Minimum sample size required to accept/reject a submitted lot for a given acceptance number with producer's risk $p^{*}$ using Poisson approximation

\begin{tabular}{|c|c|c|c|c|c|c|c|c|c|}
\hline \multirow[b]{2}{*}{$1-p^{*}$} & \multirow[b]{2}{*}{$r$} & \multicolumn{7}{|c|}{$t / \sigma$} & \multirow[b]{2}{*}{4.712} \\
\hline & & 0.628 & 0.942 & 1.257 & 1.571 & 2.356 & 3.141 & 3.927 & \\
\hline \multirow[t]{11}{*}{0.90} & 0 & 29 & 11 & 7 & 5 & 4 & 3 & 3 & 3 \\
\hline & 1 & 49 & 18 & 11 & 8 & 6 & 5 & 5 & 5 \\
\hline & 2 & 67 & 25 & 15 & 11 & 8 & 7 & 7 & 6 \\
\hline & 3 & 84 & 31 & 18 & 14 & 10 & 9 & 8 & 8 \\
\hline & 4 & 100 & 37 & 22 & 16 & 12 & 10 & 10 & 9 \\
\hline & 5 & 116 & 42 & 25 & 19 & 13 & 12 & 11 & 11 \\
\hline & 6 & 132 & 48 & 29 & 21 & 15 & 13 & 12 & 12 \\
\hline & 7 & 148 & 54 & 32 & 24 & 17 & 15 & 14 & 13 \\
\hline & 8 & 163 & 59 & 35 & 26 & 19 & 16 & 15 & 15 \\
\hline & 9 & 178 & 65 & 38 & 29 & 20 & 18 & 17 & 16 \\
\hline & 10 & 193 & 70 & 42 & 31 & 22 & 19 & 18 & 17 \\
\hline \multirow[t]{11}{*}{0.95} & 0 & 38 & 14 & 8 & 6 & 5 & 4 & 4 & 4 \\
\hline & 1 & 60 & 22 & 13 & 10 & 7 & 6 & 6 & 6 \\
\hline & 2 & 79 & 29 & 17 & 13 & 9 & 8 & 8 & 7 \\
\hline & 3 & 97 & 36 & 21 & 16 & 11 & 10 & 9 & 9 \\
\hline & 4 & 115 & 42 & 25 & 19 & 13 & 12 & 11 & 10 \\
\hline & 5 & 132 & 48 & 29 & 21 & 15 & 13 & 12 & 12 \\
\hline & 6 & 149 & 54 & 32 & 24 & 17 & 15 & 14 & 13 \\
\hline & 7 & 165 & 60 & 36 & 26 & 19 & 16 & 15 & 15 \\
\hline & 8 & 181 & 66 & 39 & 29 & 21 & 18 & 17 & 16 \\
\hline & 9 & 197 & 72 & 42 & 32 & 22 & 20 & 18 & 18 \\
\hline & 10 & 213 & 77 & 46 & 34 & 24 & 21 & 20 & 19 \\
\hline \multirow[t]{11}{*}{0.99} & 0 & 58 & 21 & 13 & 10 & 7 & 6 & 6 & 6 \\
\hline & 1 & 83 & 31 & 18 & 14 & 10 & 9 & 8 & 8 \\
\hline & 2 & 106 & 39 & 23 & 17 & 12 & 11 & 10 & 10 \\
\hline & 3 & 126 & 46 & 27 & 20 & 14 & 13 & 12 & 11 \\
\hline & 4 & 146 & 53 & 31 & 23 & 17 & 15 & 14 & 13 \\
\hline & 5 & 164 & 60 & 35 & 26 & 19 & 16 & 15 & 15 \\
\hline & 6 & 183 & 66 & 39 & 29 & 21 & 18 & 17 & 16 \\
\hline & 7 & 201 & 73 & 43 & 32 & 23 & 20 & 19 & 18 \\
\hline & 8 & 218 & 79 & 47 & 35 & 25 & 22 & 20 & 20 \\
\hline & 9 & 235 & 85 & 51 & 38 & 27 & 23 & 22 & 21 \\
\hline & 10 & 252 & 92 & 54 & 40 & 29 & 25 & 23 & 23 \\
\hline
\end{tabular}

